\title{
Transplante de fígado em nosso meio. A evolução de um procedimento estratégico institucional
}

\section{Liver Transplantation in Ribeirão Preto. The evolution of a strategic Institutional procedure}

Orlando de Castro e Silva', Fernanda Fernandes Souza², Andreza Côrrea Teixeira², Ênio David Mente², Ajith Kumar Sankarankutty ${ }^{3}$

A realização do Transplante de Fígado em um Hospital no Brasil demonstra, além da complexa estrutura Institucional, a vontade inabalável de pessoas e a riqueza organizacional do nosso Sistema Único de Saúde

No segundo semestre de 1999, demos início ao Programa de Transplantes de Fígado no Hospital das Clínicas da Faculdade de Medicina de Ribeirão Preto da Universidade de São Paulo (HCFMRP-USP), após o credenciamento tanto do hospital como da equipe médica especializada, conforme a Portaria $\mathrm{n}^{\circ} 812$ de 29 de dezembro de 1999. O primeiro paciente foi então inscrito na fila de espera e naquela época a alocação do órgão para transplante de fígado se dava pelo critério cronológico. Assim, os pacientes incluídos na lista de espera do HCFMRP-USP ocuparam posições posteriores aos dos pacientes previamente inscritos pelas equipes da Unicamp e de São José do Rio Preto, uma vez que essas equipes tinham sido credenciadas, antes de nós, em meados da década de 1990.
No ano seguinte, 2000, em editorial nesta revista, comentava sobre toda a expectativa do início do programa de transplante de fígado do HCFMRP-USP como verdadeiro desafio institucional. ${ }^{1}$ A cidade de Ribeirão Preto e as cidades vizinhas que a têm como referência para tratamento médico, o Hospital das Clínicas e a Faculdade de Medicina de Ribeirão Preto não podiam ficar à margem de um processo necessário para tratamento com possibilidade de cura para doença hepática terminal, mundialmente realizado, com resultados satisfatórios; além de propiciar avanços importantes acadêmicos para a instituição que o realiza. A realização do transplante de fígado em um hospital no Brasil demonstra, além da complexa estrutura Institucional, a vontade inabalável de pessoas e a riqueza organizacional do nosso Sistema Único de Saúde. Democraticamente, todo cidadão brasileiro tem acesso gratuito ao transplante de fígado. Nada mais é que um dever do Estado e um direito do cidadão, conforme reza a Constituição Nacional vigente.

Nesse cenário, realizamos o primeiro transplan-
1. Professor Titular da Universidade de São Paulo e Coordenador do Grupo Integrado de Transplante de Fígado do Hospital das Clínicas da Faculdade de Medicina de Ribeirão Preto USP

2. Médicos Assistentes do Grupo Integrado de Transplante de Fígado do Hospital das Clínicas da Faculdade de Medicina de Ribeirão Preto - USP

3. Professor Doutor do Grupo Integrado de Transplante de Fígado do Hospital das Clínicas da Faculdade de Medicina de Ribeirão Preto - USP
Correspondência: Prof. Dr. Orlando de Castro e Silva Grupo Integrado de Transplante de Fígado Hospital das Clínicas da FMRP- USP 14048-900 - Ribeirão Preto-SP,

Artigo recebido em 02/12/2009 Aprovado em 18/12/2009 
te de fígado em $1^{\circ}$ de maio de $2001 .^{2,3}$ A partir daí, até o primeiro semestre de 2004, realizamos 17 transplantes apenas, pois como dito, os pacientes das outras duas equipes atuantes no interior de São Paulo tinham prioridade pois estavam nos primeiros lugares na lista de espera, uma vez que se usava o critério cronológico, para alocação do órgão. Adicionalmente, neste período, o Programa teve que ser suspenso por um ano, sobretudo por falta de recursos humanos.Esses fatos impediam o desenvolvimento pleno do programa e causava desmotivação em muitos dos membros da Equipe. Foram tempos difíceis mas que haveria de passar.

De fato, vencidas as dificuldades, as atividades foram reiniciadas em meados de 2004 e, apesar de muitas limitações ainda existentes, conseguimos conduzir o Programa em condições harmônicas. Em fins de 2004 aumentamos substancialmente a Equipe e a partir de então, com um trabalho otimizado e multiprofissonal em sua essência,o Programa caminhou com crescimento exponencial ao longo dos anos, até o final deste ano, como é possível observármos na figura 1 .

Em meados de 2006, após quase um ano de debates acalorados em todo Brasil, e em todos os níveis, implantou-se o critério de gravidade MELD (Model for End stage Liver Diseases) para alocação de órgaõs para transplante de fígado no Brasil confor- me a Portaria Ministerial $\mathrm{n}^{\circ}-1.160$, de 29 de maio de 2006. ${ }^{4,5}$

O MELD é modelo matemático em que se usa em uma fómula logarítmica os valores da creatinina sérica, bilirrubina total sérica e o INR (international normalized ratio). Além do valor absoluto do MELD, ajustes foram feitos para atribuir uma pontuação complementar para algumas situações denominadas como especiais pelo Ministério da Saúde, tais como carcinoma hepatocelular, doenças metabólicas, polineuropatia amiloidótica familiar, dentre outras. Dessa forma, o número de procedimentos aumentou, principalmente em função das situações especiais, após rigorosa avaliação pelas câmaras de análises técnicas estaduais ou nacional quando necessário.

Apesar de ter sido um avanço na alocação de órgãos, este critério ainda não contempla algumas situações graves em que o escore MELD é baixo, mas o paciente tem uma qualidade de vida ruim, por exemplo pacientes com cirrose hepática e complicações como: encefalopatia crônica, ascite refratária e hemorragia digestiva alta. Tal fato é ainda uma realidade de difícil resolução nos ambulatórios de hepatologia e transplante de fígado

Adicionalmente, deve ser considerado um aumento real no número de doações. As famílias brasileiras, melhor conscientizadas, doam seus órgãos hoje mais que no passado e os hospitais, longe ainda do ideal, estão se equipando melhor para atendimento

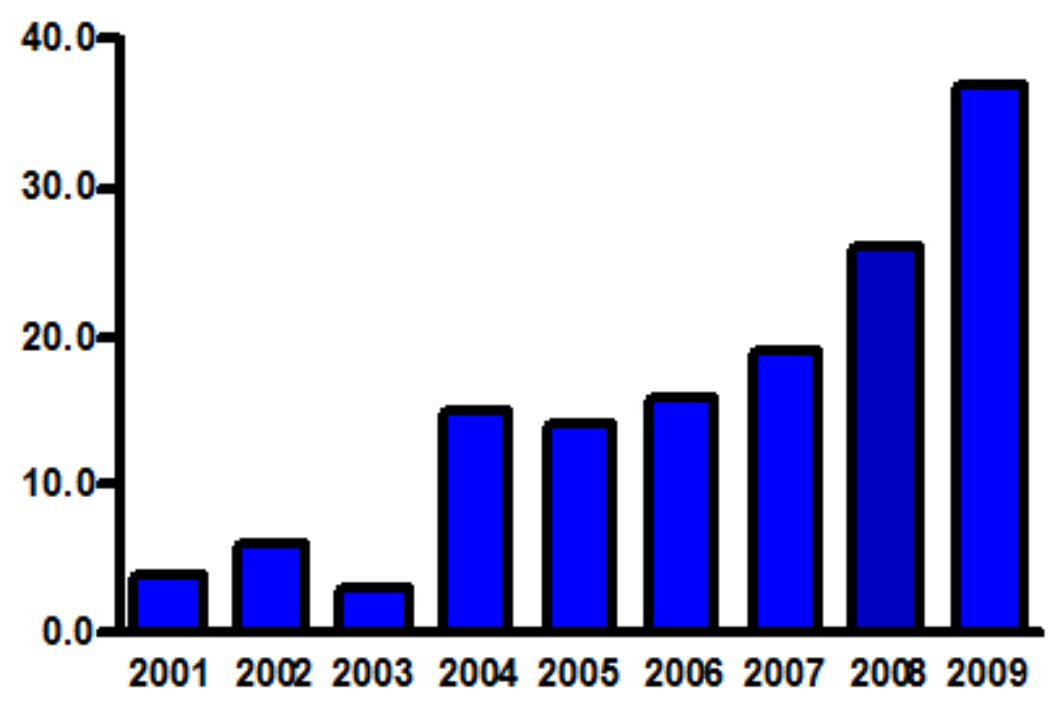

Figura 1. Número de transplantes de fígado realizados no Hospital das Clínicas da Faculdade de Medicina de Ribeirão Preto da Universidade de São Paulo, discriminados ano a ano, de 2001 a 2009 
mais adequado dos doadores potenciais e efetivos, gerando aumento na quantidade e na qualidade de órgãos para transplante, de um modo geral.

Em 2009 realizamos trinta e seis transplantes de fígado no HCFMRP-USP, número que deve ser mantido no próximo ano, para sucessivamente atingirmos os possíveis 40 transplantes de fígado ao ano, porém com idealização e potencial de serem realizados cinco transplantes por mês (60 ao ano). A seguir descreveremos os motivos mais freqüentes pelos quais esse objetivo ainda não foi alcançado: manutenção do doador inadequadada para transplantes; impossibilidade de realização de dois transplantes simultaneamente; receptores sem condições clínicas para realização do transplante no momento da doação; problemas infraestruturais do hospital no momento da doação. Enfim, são muitas as causas de não aproveitamento de órgãos em geral e do fígado, em particular, e que, invariavelmente, ocorrem na maioria dos serviços de transplantes mundo a fora. Cabe a nós, equipe e direção do hospital, tomarmos as dignas providências para que seja possível o crescimento. Os números da figura 1 precisam continuar num crescimento exponencial.

Em janeiro de 2005, inaugurou-se a Unidade Especial de Transplante de Fígado (UTF). Diante do aumento do número de transplantes, tornava-se necessário termos um local para concentrar os pacientes, tanto os transplantados como os da lista de espera que freqüentemente apresentam complicações e necessitam de internação. Com a UTF, tornou-se possível oferecer a esse grupo de pacientes um atendimento individualizado, com equipe multidisciplinar especializada.

Entretanto, quatro anos após a abertura, a UTF necessita de ampliação, pois encontra-se insuficiente, tanto no que tange à infra-estrutura quanto aos recursos humanos, para suprir a demanda a qual lhe é solicitada.

A sobrevida, em torno de $70 \%$ ao ano, mostra a eficácia do Programa e a necessidade de sua expansão. A expectativa do início do transplante pediátrico de fígado para o próximo ano e o apoio logístico a outros tipos de transplantes na Instituição geram entusiasmo na equipe e surgem novas necessidades que, passo a passo, precisam ser contempladas.

O transplante de fígado, por sua complexidade, gera recursos para o hospital, visto que, além de ser um procedimento "estratégico" para o SUS, é extrateto, ou seja, quanto mais se faz, mais ganha o hospital. Este procedimento absorve tecnologia e a transfere a outros setores da Instituição. Melhora a assistência, melhora o ensino e gera conhecimento. Acreditamos que nossa comunidade tenha percebido isto nestes quase nove anos de transplante de fígado no HCRMRP-USP.

Esperamos que em maio de 2011 possamos comemorar nossos 200 transplantes de fígado, nos mantendo entre os cinco melhores serviços de transplante, deste órgão, do Estado de São Paulo, em quantidade e qualidade de trabalho.É um grande desafio, não só para nossa Equipe como também para o Hospital das Clínicas da Faculdade de Medicina de Ribeirão Preto da Universidade de São Paulo.

\section{Referências Bibliográficas}

1. Castro e Silva O, Martinelli AL. Editorial.Transplante de fígado.Uma realidade no Hospital das Clínicas da FMRP-USP para o ano 2001. Medicina (Ribeirão Preto) 2000;33: 373-6.

2. Castro e Silva $O$.Editorial Primeiro transplante de fígado em Ribeirão Preto. Medicina (Ribeirão Preto) 2001; 34:119-20.

3. Franca AVC, Martinelli ALC, Sankarankutty AK, Rizzo CC, Castro e Silva O. Tranplante de fígado: relato do primeiro caso realizado no Hospital das Clínicas da Faculdade de Medicina de Ribeirão Preto da Universidade de São Paulo. Medicina (Ribeirão Preto) 2001; 34:194-9.

4. Teixeira AC, Souza FF, Mota GA, Martinelli ALC, Sankarankutty AK, Castro e Silva O. Liver transplantation: expectation with MELD score for liver allocation in Brazil. Acta Cir Bras. [serial on the Internet] 2006; 21 Suppl 1. Available from URL: http:// www.scielo.br/acb

5. Brasil. Ministério da Saúde. Critério para distribuição de fígado doador cadáver para transplante. Portaria no. 1.160 de 29 de maio de 2006, publicada no Diário Oficial da União, no 103 de 31 de maio de 2006. 